\title{
Microwave Photonics
}

\author{
Borja Vidal, ${ }^{1}$ Nathan J. Gomes, ${ }^{2}$ Tadao Nagatsuma, ${ }^{3}$ and Thomas E. Darcie ${ }^{4}$ \\ ${ }^{1}$ Nanophotonics Technology Center, Polytechnic University of Valencia, 46022 Valencia, Spain \\ ${ }^{2}$ Broadband and Wireless Communications Group, University of Kent, Canterbury CT2 7NT, UK \\ ${ }^{3}$ Department of Systems Innovation, Graduate School of Engineering Science, Osaka University, 1-1 Yamadaoka, \\ Suita, Osaka 565-0871, Japan \\ ${ }^{4}$ Department of Electrical and Computer Engineering, University of Victoria, Victoria, BC, Canada V8W $3 P 6$
}

Correspondence should be addressed to Borja Vidal, bvidal@dcom.upv.es

Received 22 October 2012; Accepted 22 October 2012

Copyright (c) 2012 Borja Vidal et al. This is an open access article distributed under the Creative Commons Attribution License, which permits unrestricted use, distribution, and reproduction in any medium, provided the original work is properly cited.

The continuous evolution of photonic technology in the last four decades has transformed the world allowing the development of the Internet, social networks, video on demand as well as considerable advances in medicine and industry. Photonic components can be also applied to the generation, transmission, and processing of microwave and millimeter-wave signals and benefit from the outstanding features of fiber optics. From early work in the late $70 \mathrm{~s}$, this field, known as microwave photonics, has expanded considerably, producing a number of applications and attracting the interest of a wide range of researchers. These applications include radio-over-fiber links for mobile and satellite systems, remote feeding and control of microwave antennas, photonic-assisted analog-to-digital conversion, and systems and techniques for optical signal processing.

This special issue is intended to document some of the recent advances in the field. The contributions represent a diverse sample of current work on the field.

The paper entitled "Photonic technologies for millimeterand submillimeter-wave signals" is a review paper presenting recent advances in the use of optical components for the generation, transmission, and processing of signals in the upper region of the millimeter-wave (beyond $30 \mathrm{GHz}$ ). This region of the radio spectrum is hardly used for practical applications due to the difficulty in dealing with signals of this frequency. However photonics can provide a feasible alternative for the applications in this band.

The paper entitled "Photonic heterodyne pixel for imaging arrays at microwave and MM-wave frequencies" presents a $3 \times$ 3 imaging array based on a semiconductor optical amplifier heterodyne receiver.

The paper entitled "Fast optical beamforming architectures for satellite-based applications" compares several architectures for fast optical beam forming of phased array antennas with particular application to space applications. Measurements of a fast beamformer without beam squint are provided between 4 and $8 \mathrm{GHz}$.

In the paper entitled " $\mathrm{X}$-Cut $\mathrm{LiNbO}{ }_{3}$ optical modulators using gap-embedded patch-antennas for wireless-over-fiber systems," an $\mathrm{x}$-cut $\mathrm{LiNbO}_{3}$ modulator integrated with gapembedded patch-antennas is proposed for wireless-overfiber applications. It showed an enhancement of $6 \mathrm{~dB}$ in comparison to z-cut $\mathrm{LiTaO}_{3}$ devices.

Finally, "Continuous operation of a Bragg diffraction type electrooptic frequency shifter at $16 \mathrm{GHz}$ with $65 \%$ efficiency" demonstrated for the first time the continuous operation of a Bragg diffraction waveguide-based electrooptic frequency shifter using a $16 \mathrm{GHz}$ modulation signal. It provides an improved factor of 11 compared to a conventional bulk device.

The Guest Editors hope that this special issue will contribute to advance the research in this field as well as to the deployment of novel industrial applications for microwave photonic products. We thank the contributors for their report of significantly new results and the reviewers for their detailed evaluations that have strengthened the papers.

Borjo Vidal Nathan J. Gomes Tadao Nagatsuma Thomas E. Darcie 

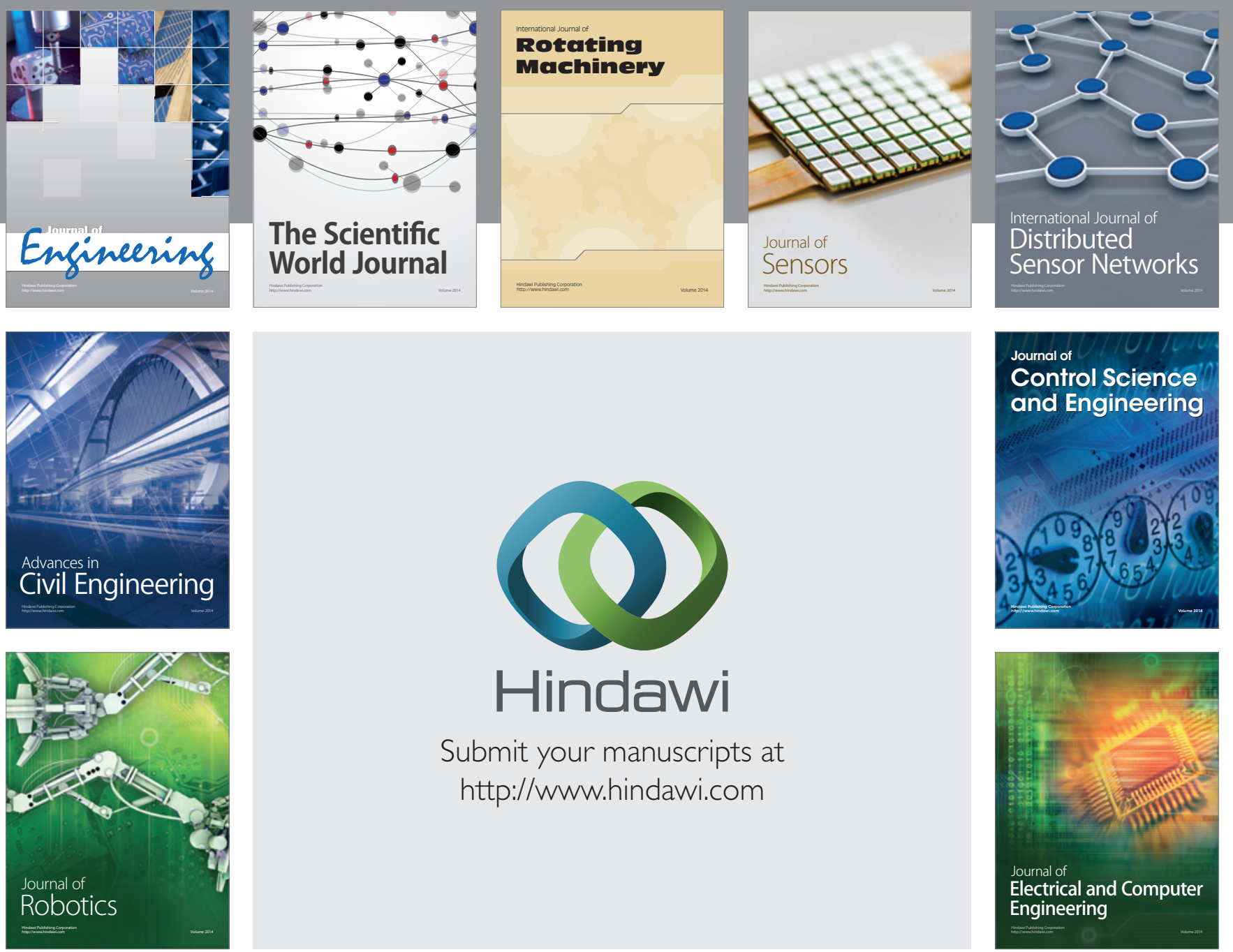

Submit your manuscripts at

http://www.hindawi.com
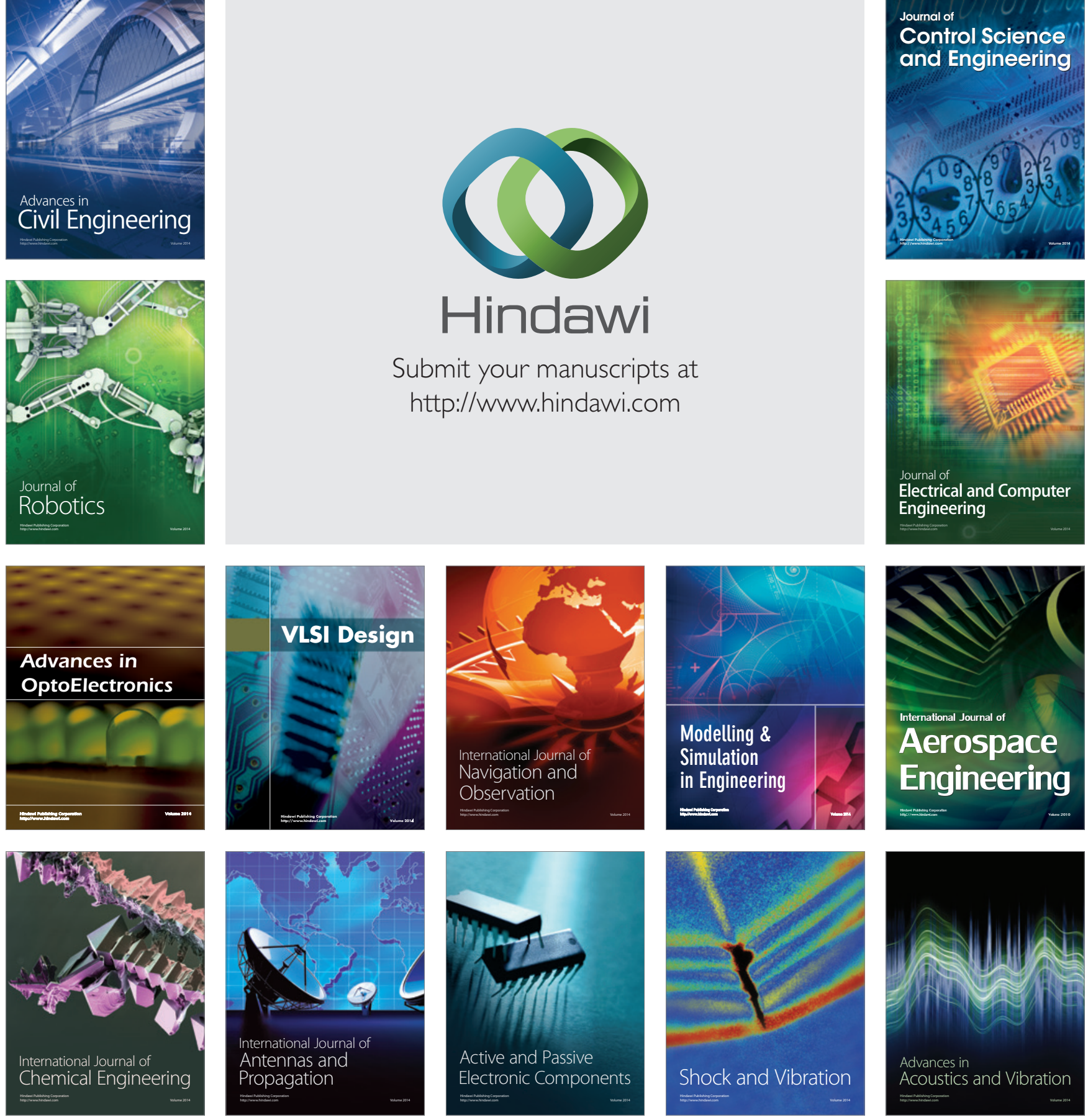